\title{
Slotted Aloha as a game with partial information
}

\author{
Eitan Altman $^{\text {a }}$, Rachid El Azouzi ${ }^{\text {a,b,*, }}$, Tania Jiménez ${ }^{\mathrm{c}}$ \\ a INRIA, 2004 Route des Lucioles, Projet Mistral, 06902 Sophia Antipolis Cote d'Azur Cedex, France \\ b LIA/CERI, Université d'Avignon, Agroparc, BP 1228, 84911 Avignon, France \\ c CESIMO, Facultad de Ingeniería, Universidad de Los Andes, Mérida, Venezuela
}

Received 13 March 2003; received in revised form 19 February 2004; accepted 25 February 2004

Available online 7 April 2004

Responsible Editor: E.K.P. Chong

\begin{abstract}
This paper studies distributed choice of retransmission probabilities in slotted ALOHA. Both the cooperative team problem as well as the noncooperative game problem are considered. Unlike some previous work, we assume that mobiles do not know the number of backlogged packets at other nodes. A Markov chain analysis is used to obtain optimal and equilibrium retransmission probabilities and throughput. We then investigate the impact of adding retransmission costs (which may represent the disutility for power consumption) on the equilibrium and show how this pricing can be used to make the equilibrium throughput coincide with the optimal team throughput.
\end{abstract}

(C) 2004 Elsevier B.V. All rights reserved.

Keywords: Slotted Aloha; Nash equilibrium; Markov chain; Pricing

\section{Introduction}

Aloha [4] and slotted Aloha [14] have long been used as random distributed medium access protocols for radio channels. They are in use in both satellite as well as cellular telephone networks for the sporadic transfer of data packets. In these protocols, packets are transmitted sporadically by various users. If packets are sent simultaneously by more than one user then they collide. After the end of the transmission of a packet, the transmitter receives the information on whether there has been a collision (and retransmission is needed) or whether it was well received. All packets involved in a collision are assumed to be corrupted and are retransmitted after some random time. We focus in this paper on the slotted Aloha (which is known to have a better achievable throughput than the unslotted version, [5]) in which time is

\footnotetext{
${ }^{*}$ Corresponding author. Address: INRIA, 2004 Route des Lucioles, Projet Mistral, 06902 Sophia Antipolis Cote d'Azur Cedex, France. Tel.: +33-492387628; fax: +33-492387971.

E-mail address: rachid.elazouzi@sophia.inria.fr (R. El Azouzi).
} 
divided into units. At each time unit a packet may be transmitted, and at the end of the time interval, the sources get the feedback on whether there was zero, one or more transmissions (collision) during the time slot. A packet that arrives at a source is immediately transmitted. Packets that are involved in a collision are backlogged and are scheduled for retransmission after a random time.

The determination of the above random time can be considered as a stochastic control problem. The information structure, however, is not a classical one: sources do not have full state information as they do not know how many packets are backlogged. Nor do they know how many packets have been involved in a collision.

We study this control problem in two different frameworks:

1. As a team problem, i.e. where there is a common goal to all nodes in the network (such as maximizing the system throughput).

2. As a problem in a noncooperative framework: each node wishes to maximize its own throughput. This gives rise to a game theoretical formulation.

Our main finding is that as the workload increases (i.e. as the packet arrival rate increases), sources become more aggressive at equilibrium in the game setting (in comparison with the team problem) and this results in a dramatic decrease in the total system's throughput. To avoid this collapse of system's throughput, we study the effect of adding a cost for transmissions and retransmissions (which can, in particular, represent the battery power cost). We show that this additional cost improves the system's performance and that an appropriate pricing can be chosen that yields an equilibrium performance that coincides with the team one.

Previous game formulations of the slotted ALOHA have been proposed in [10-12]. In the two last references, a full information game is considered, in which each user knows how many backlogged packets there are in all the network. Moreover, it is assumed in [11,12] that a packet that is to be transmitted for the first time waits for a random time in the same way as a backlogged packet. Our goal is to study the slotted Aloha avoiding these two assumptions; relaxing the assumptions allows to model more accurately the original versions of Aloha, and in particular, relaxing the first assumption allows for more distributed implementations of Aloha. In [10] it is assumed that nodes have always packets to send. Thus there is only one trivial state in the system (all nodes are backlogged) which is known to all users.

For more background on the use of stochastic control and of game theory in communication networks, see [1-3]. We note that the game formulation of our problem is similar to game formulation of retrial queues, in which customers retry to make a call after some random time if they find the line busy [7,9]. The difference is, however, that in retrial queues there are no collisions.

The structure of the paper is as follows. We begin by introducing in Section 2 the general model and formulate the team and the game problems. We provide a Markov analysis for both the team and the game problem. This analysis is used in Section 3 to numerically study and compare the properties of the team and the game solutions. The model with pricing is then introduced in Section 4 and is investigated numerically in Section 5. We end with a concluding section.

\section{Model and problem formulation}

We use a Markovian model based on [5]. We assume that there are a finite number of sources without buffers. The arrival flow of packets to source $i$ follows a Bernoulli process with parameter $q_{a}$ (i.e. at each time slot, there is a probability $q_{a}$ of a new arrival at a source, and all arrivals are independent). As long as there is a packet at a source (i.e. as long as it is not successfully transmitted) new packets to that 
source are blocked and lost. ${ }^{1}$ The arrival processes to different sources are independent. A backlogged packet at source $i$ is retransmitted with probability $q_{r}^{i}$. We shall restrict in our control and game problems to simple policies in which $q_{r}^{i}$ does not change in time. Since sources are symmetric, we shall further restrict to finding a symmetric optimal solution, that is retransmission probabilities $q_{r}^{i}$ that do not depend on $i$. We assume that if more than one source attempt transmission in a time slot, all packets are lost.

Remark 1. Other models for ALOHA have been also studied in the literature. A commonly used model is one with infinite many sources [5] with no buffers, in which the process of total number of (nonblocked) arrivals at a time slot is Poisson with parameter $\lambda$ and the process of combined transmissions and retransmissions attempts forms a Poisson process with parameter $G$. Analysis of this model shows that it has two quasi-stable operation modes (as long as $\lambda<\exp (-1)$ ), one corresponding to a congested system (in which there are many backlogged packets and many retransmissions) and one corresponding to an uncongested system (with small amount of backlogged packets). In this model, both operation points turn out to have the same throughput. In our model with finitely many sources we has also two quasi-stable operation modes but the throughput during congestion periods is lower than in the noncongested periods [5]. We also note that in the case of infinitely many nodes, retransmissions with a fixed positive probability renders the system unstable [8]. Finally, we should mention that there are also models in which not all packets involved in a collision are corrupted and lost, see [15] and references therein.

Remark 2. Quite frequently one uses the ALOHA protocol for sporadic transmissions of signaling packets such as packets for making reservation for a dedicated channel for other transmissions (that do not use ALOHA), see e.g. the description of the SPADE on demand transmission protocol for satellite communications in [16]. In the context of signaling, it is natural to assume that a source does not start generating a new signaling packet (e.g. a new reservation) as long as the current signaling packet is not transmitted. In that case, the process of attempts to retransmit a new packet from a source after the previous packet has been successfully transmitted coincides with our no buffer model.

We shall use as the state of the system the number of backlogged nodes (or equivalently, of backlogged packets) at the beginning of a slot, and denote it frequently with $n$. For any choice of values $q_{r}^{i} \in(0,1]$, the state process is a Markov chain that contains a single ergodic chain (and possibly transient states as well). Define $\mathbf{q}_{\mathbf{r}}$ to be the vector of retransmission probabilities for all users (whose $j$ th entry is $q_{r}^{j}$ ). Let $\pi\left(\mathbf{q}_{\mathbf{r}}\right.$ ) be the corresponding vector of steady state probabilities where its $n$th entry, $\pi_{n}\left(\mathbf{q}_{\mathbf{r}}\right)$, denotes the probability of $n$ backlogged nodes. When all entries of $\mathbf{q}_{\mathbf{r}}$ are the same, say $q$, we shall write (with some abuse of notation) $\pi(q)$ instead of $\pi\left(\mathbf{q}_{\mathbf{r}}\right)$.

We introduce further notation. Assume that there are $n$ backlogged packets, and all use the same value $q_{r}$ as retransmission probability. Let $Q_{r}(i, n)$ be the probability that $i$ out of the $n$ backlogged packets retransmit at the slot. Then

$$
Q_{r}(i, n)=\left(\begin{array}{c}
n \\
i
\end{array}\right)\left(1-q_{r}\right)^{n-i}\left[q_{r}\right]^{i}
$$

\footnotetext{
${ }^{1}$ In considering the number of packets in the system, this assumption is equivalent to saying that a source does not generate new packets as long as a previous packet is not successfully transmitted.
} 
Assume that $m$ is the number of nodes and let $Q_{a}(i, n)$ be the probability that $i$ unbacklogged nodes transmit packets in a given slot (i.e. that $i$ arrivals occurred at nodes without backlogged packets). Then

$$
Q_{a}(i, n)=\left(\begin{array}{c}
m-n \\
i
\end{array}\right)\left(1-q_{a}\right)^{m-n-i}\left[q_{a}\right]^{i}
$$

Let $Q_{r}(1,0)=0$ and $Q_{a}(1, m)=0$. $[5]:$

In case all nodes use the same value $q$ for $q_{r}$, the transition probabilities of the Markov chain are given by

$$
P_{n, n+i}(q)=\left\{\begin{array}{l}
Q_{a}(i, n), \quad 2 \leqslant i \leqslant m-n, \\
Q_{a}(1, n)\left[1-Q_{r}(0, n)\right], \quad i=1, \\
Q_{a}(1, n) Q_{r}(0, n)+Q_{a}(0, n)\left[1-Q_{r}(1, n)\right], \quad i=0, \\
Q_{a}(0, n) Q_{r}(1, n), \quad i=-1 .
\end{array}\right.
$$

The system throughput (defined as the sample average of the number of packets that are successfully transmitted) is given almost surely by the constant

$$
\operatorname{thp}(q)=\sum_{n=1}^{m} \pi_{n}(q)\left[P_{n, n-1}(q)+Q_{a}(1, n) Q_{r}(0, n)\right]+\pi_{0}(q) Q_{a}(1,0)=q_{a} \sum_{n=0}^{m} \pi_{n}(q)(m-n) .
$$

Note: the first equality follows from the fact that if the state at the beginning of the slot is $n>0$ then there is a departure of a backlogged packet during that slot with probability $P_{n, n-1}(q)$, and of a new arriving packet with probability $Q_{a}(1, n) Q_{r}(0, n)$; Moreover, if the state is 0 then there is a departure with probability $Q_{a}(1,0)$. The second equality simply expresses the expected number of arrivals at a time slot (which actually enter the system), which should equal to the expected number of departures (and thus the throughput) at stationary regime.

The team problem is therefore given as the solution of the optimization problem:

$$
\max _{q} \operatorname{thp}(q) \quad \text { s.t. }\left\{\begin{array}{l}
\pi(q)=\pi(q) P(q), \\
\pi_{n}(q) \geqslant 0, \quad n=0, \ldots, m, \\
\sum_{n=0}^{m} \pi_{n}(q)=1 .
\end{array}\right.
$$

A solution to the team problem can be obtained by computing recursively the steady state probabilities, as in Problem 4.1 in [5], and thus obtain an explicit expression for $\operatorname{thp}(q)$ as a function of $q$.

Singularity at $q=0$. The only point where $P$ does not have a single stationary distribution is at $q=0$, where it has two absorbing states: $n=m$ and $n=m-1$. All other states are transient (for any $q_{a}>0$ ), and the probability to end at one of the absorbing states depend on the initial distribution of the Markov chain. We note that if the state $m-1$ is reached then the throughput is $q_{a}$ w.p.1, where as if the state $m$ is reached then the throughput equals 0 . It is thus a deadlock state. For $q_{a}>0$ and $q_{r}=0$, the deadlock state is reached with positive probability from any initial state other than $m-1$. We shall therefore exclude $q_{r}=0$ and optimize only on the range $\epsilon \leqslant q_{r} \leqslant 1$. We choose throughout the paper $\epsilon=10^{-4}$.

Existence of a solution. The steady state probabilities $\pi(q)$ are continuous over $0<q \leqslant 1$. Since this is not a close interval, a solution need not exist. However, as we restrict to the closed interval $q \in[\epsilon, 1]$ where

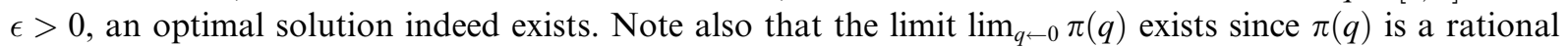
function of $q$ at the neighborhood of zero. Therefore for any $\delta>0$, there exists some $q>0$ which is $\delta$ optimal. ( $q^{*}>0$ is said to be $\delta$-optimal if it satisfies $\operatorname{thp}\left(q^{*}\right) \geqslant \operatorname{thp}(q)-\delta$ for all $q \in(0,1]$.)

Next, we formulate the game problem. For a given policy vector $\mathbf{q}_{\mathbf{r}}$ of retransmission probabilities for all users (whose $j$ th entry is $q_{r}^{j}$ ), define $\left(\left[\mathbf{q}_{\mathbf{r}}\right]^{-i}, \hat{q}_{r}^{i}\right)$ to be a retransmission policy where user $j$ retransmits at a slot with probability $q_{r}^{j}$ for all $j \neq i$ and where user $i$ retransmits with probability $\hat{q}_{r}^{i}$. Each user $i$ seeks to 
maximize his own throughput thp $\mathrm{p}_{i}$. The problem we are interested in is then to find a symmetric equilibrium policy $\mathbf{q}_{\mathbf{r}}^{*}=\left(q_{r}, q_{r}, \ldots, q_{r}\right)$ such that for any user $i$ and any retransmission probability $q_{r}^{i}$ for that user,

$$
\operatorname{thp}_{i}\left(\mathbf{q}_{\mathbf{r}}^{*}\right) \geqslant \operatorname{thp}_{i}\left(\left[\mathbf{q}_{\mathbf{r}}^{*}\right]^{-i}, q_{r}^{i}\right) .
$$

Since we restrict to symmetric $\mathbf{q}_{\mathrm{r}}^{*}$, we shall also identify it (with some abused of notation) with the actual transmission probability (which is the same for all users). Next we show how to obtain an equilibrium policy. We first note that due to symmetry, to see whether $\mathbf{q}_{\mathbf{r}}^{*}$ is an equilibrium it suffices to check (3) for a single player. We shall thus assume that there are $m+1$ users all together, and that the first $m$ users retransmit with a given probability $\mathbf{q}_{\mathbf{r}}^{-(m+1)}=\left(q^{o}, \ldots, q^{o}\right)$ and user $m+1$ retransmits with probability $q_{r}^{(m+1)}$. Define the set

$$
\mathscr{2}^{m+1}\left(\mathbf{q}_{\mathbf{r}}^{\mathbf{o}}\right)=\operatorname{argmax}_{q_{r}^{(m+1)} \in[\epsilon, 1]}\left(\operatorname{thp}_{m+1}\left(\left[\mathbf{q}_{\mathbf{r}}^{\mathbf{0}}\right]^{-(m+1)}, q_{r}^{(m+1)}\right)\right),
$$

where $\mathbf{q}_{\mathbf{r}}^{\mathbf{0}}$ denotes (with some abuse of notation) the policy where all users retransmit with probability $q_{r}^{o}$, and where the maximization is taken with respect to $q_{r}^{(m+1)}$. Then $q_{r}^{*}$ is a symmetric equilibrium if

$$
q_{r}^{*} \in \mathscr{2}_{r}^{m+1}\left(q_{r}^{*}\right) .
$$

To compute thp $\mathrm{p}_{m+1}\left(\left[\mathbf{q}_{\mathbf{r}}^{\mathbf{o}}\right]^{-i}, q_{r}^{i}\right)$, we introduce again a Markov chain with a two dimensional state. The first state component corresponds to the number of backlogged packets among the users $1, \ldots, m$, and the second component is the number of backlogged packets (either 1 or 0 ) of user $m+1$. The transition probabilities are given by

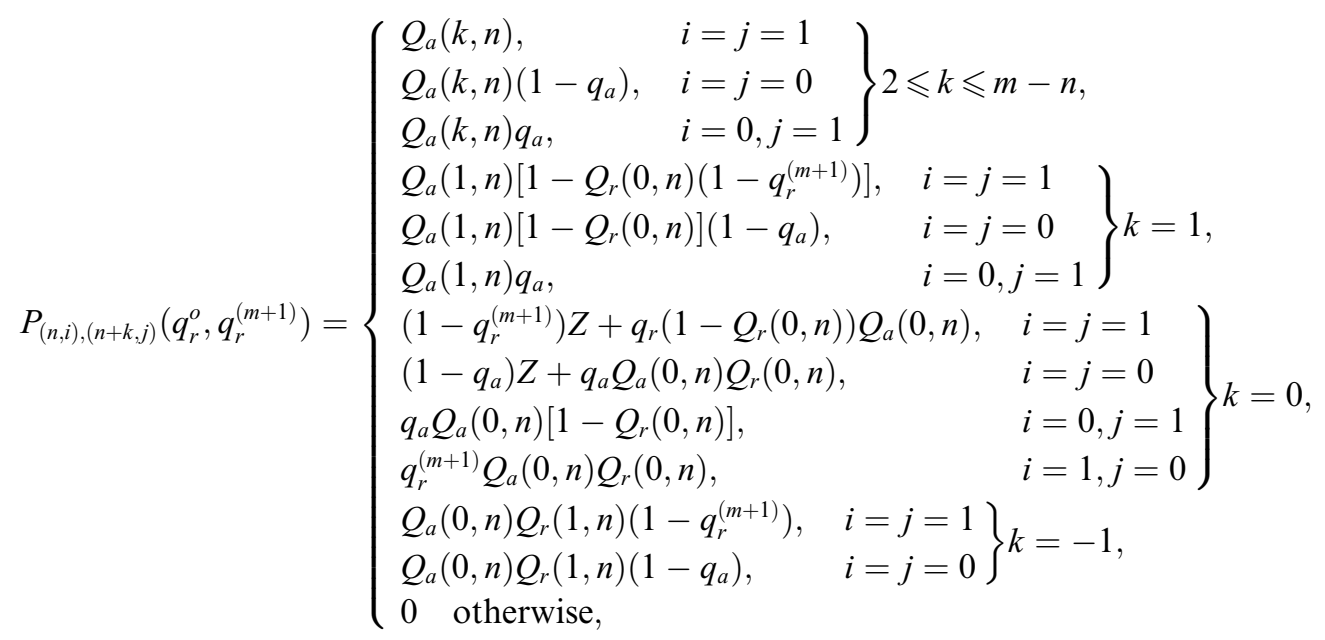

where $Z=\left(Q_{a}(1, n) Q_{r}(0, n)+Q_{a}(0, n)\right)\left[1-Q_{r}(1, n)\right]$ and where $Q_{a}$ and $Q_{r}$ are given in (1) and (2), respectively (with $q_{r}^{o}$ replacing $q_{r}$ ).

The throughput of user $m+1$ is given by

$$
\operatorname{thp}_{m+1}\left(\left[\mathbf{q}_{\mathbf{r}}^{\mathbf{0}}\right]^{-(m+1)}, q_{r}^{(m+1)}\right)=q_{a} \sum_{n=0}^{m} \pi_{n, 0}\left(\left[q_{r}^{o}\right]^{-(m+1)}, q_{r}^{(m+1)}\right) .
$$

\section{Numerical investigation}

In this section we shall obtain the retransmission probabilities which solve the team and the game problem. We investigate their dependence and the dependence of the throughput that they imply on the arrival probabilities $q_{a}$ and on the number of mobiles. 
Figs. 1 and 2 provide the total throughput and optimal retransmission probabilities $q_{r}$ for $m=2, m=10$ and $m=50$ for the team problem, as a function of the arrival probability $q_{a}$. We see that in heavy traffic, the throughput decreases when the number of mobiles increases. Also, we observe that the optimal retransmission policy is more and more small when the arrival probability increases or the number of mobiles increases: as the system becomes more congested (larger arrival probability or large number of mobiles) the transmission probability decreases so as to counter expected collisions. But for light traffic, we observe that the slotted Aloha is very efficient when the number of mobiles is large: in that regime, the optimal throughput achieved increases as the number of mobile increases.

The intuitive reason that the team optimal retransmission probabilities are close to 0 when arrival probabilities are close to one is that if a mobile finds all other mobiles backlogged then it can transmit for very long time all its packets at a rate of a almost one packet per slot, without fearing collisions. Since its arrival probabilities are close to one, then throughput is not wasted during such periods. (Note however that a throughput close to 1 cannot be achieved since with some nonnegligible probability, all mobiles will be backlogged during long periods when retransmission probabilities are very low.) The behavior we see could remind of CDMA systems in which best performance is sometime achieved by "time-sharing" the access between users in order to decrease interference [13].

Next, we show in Figs. 3 and 4 the total optimal throughput versus the number of mobiles for some fixed arrival probabilities $\left(q_{a}=0.7,0.8,0.9\right)$. In Fig. 3 we observe that the optimal throughput converges to some value when the number of mobiles goes to infinity, and convergence is faster when the arrival probability $q_{a}$ is larger. In fact, for heavy traffic with large number of mobiles, the optimal retransmission probability is seen to be $\epsilon$. Thus, the steady steady probabilities $\pi$ are then close to $\pi_{m}=1 / 2, \pi_{m-1}=1 / 2$ and $\pi_{n}=0$ $\forall n<m-1$. Hence the total throughput becomes $q_{a} / 2$. If we look of the value of the throughput on the $y$ axis of Fig. 3 we observe that the throughput indeed converges to 0.35 (resp. 0.4, 0.45) for $q_{a}=0.7$ (resp. $q_{a}=0.8, q_{a}=0.9$ ).

Now, we present the results we obtain when we use the game problem. Figs. 5 and 6 show total throughput at equilibrium (obtained by multiplying the expression in Eq. (4) by the number of mobiles) and the retransmission probability at equilibrium as a function of the arrival probability for the game scenario. We see that for game problem, in contrast to team problem, the equilibrium retransmission becomes more and more aggressive as the arrival probability increases or the number of mobiles increases which explains the dramatic decrease in the system's throughput. Moreover, the equilibrium retransmission quickly increases to 1 when the number of mobiles increases. In particular the throughput is zero when $m>5$ for each arrival probability. In conclusion, the game solution is very inefficient for heavy traffic, and even for light traffic it becomes inefficient when the number of mobiles is larger than five.

We note that a similar aggressive behavior at equilibrium has been observed in [6] in the context of flow control by several competing users that share a common drop tail buffer. However in that context, the most aggressive behavior (of transmission at maximum rate) is the "equilibrium" solution for any arrival rate, and not just at high rates as in our case. We may thus wonder why retransmission probabilities of 1 are not an equilibrium in our slotted Aloha problem (in the case of light traffic). An intuitive reason could be that if a mobile deviates and retransmits with probability one, (while other continue to retransmit with the equilibrium probability $q^{*}<1$ ) the total congestion in the system (i.e. the number of backlogged mobiles) increases; this provokes more retransmissions from other mobiles which then causes sufficiently more collisions of packets from the deviating mobile so as to cause a decrease in its throughput.

\section{Adding costs for retransmissions}

In this section we consider the problem where there is an extra cost $\theta$ per each transmission and retransmission. This can represent the disutility for the consumption of battery energy, which is a scarce 


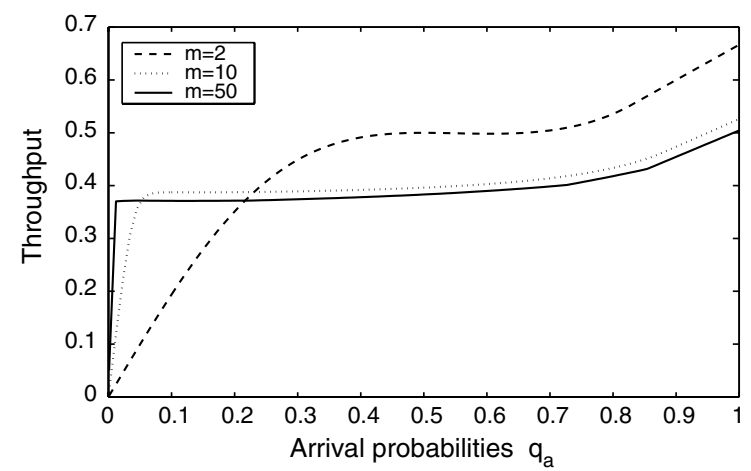

Fig. 1. Optimal throughput for the team case as a function of the arrival probabilities $q_{a}$ for $m=2,10,50$.

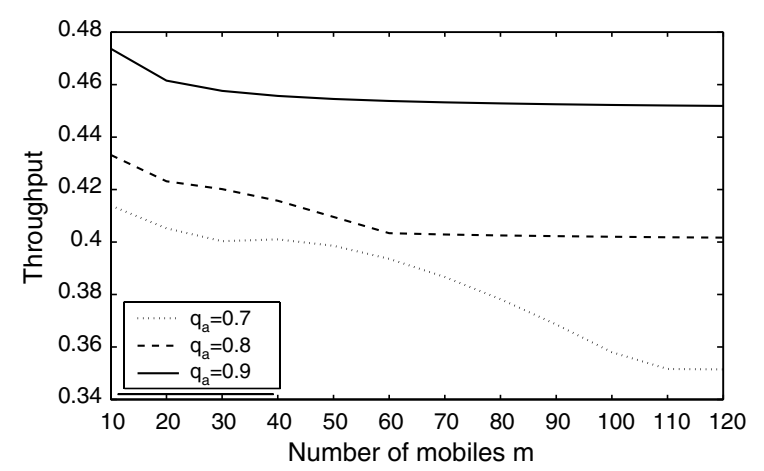

Fig. 3. Optimal throughput for the team case as a function of the number of mobiles $m$ for $q_{a}=0.7,0.8,0.9$.

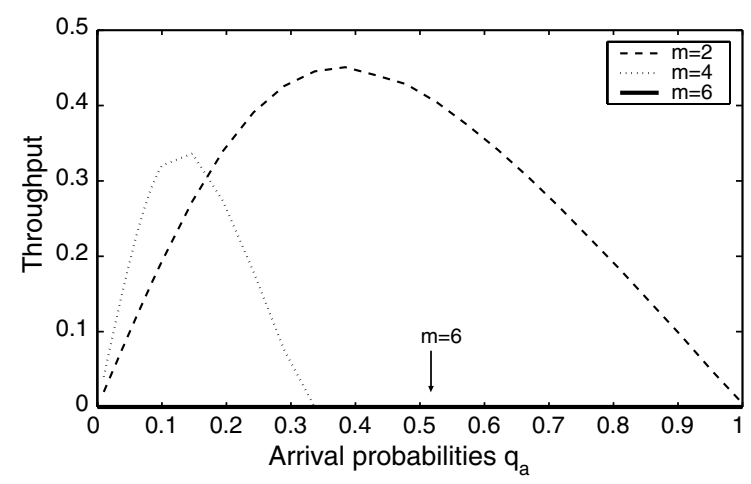

Fig. 5. Optimal throughput for the game case as a function of the arrival probabilities $q_{a}$ for $m=2,4,6$.

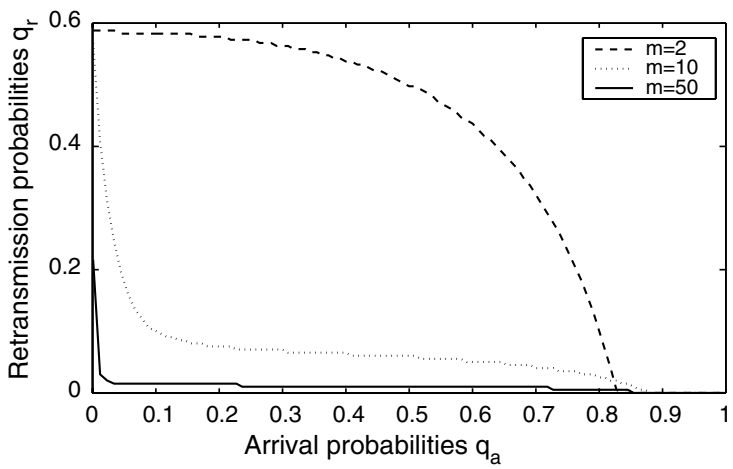

Fig. 2. The optimal retransmission probabilities in the team case as a function of the arrival probabilities $q_{a}$ for $m=2,10$, 50 .

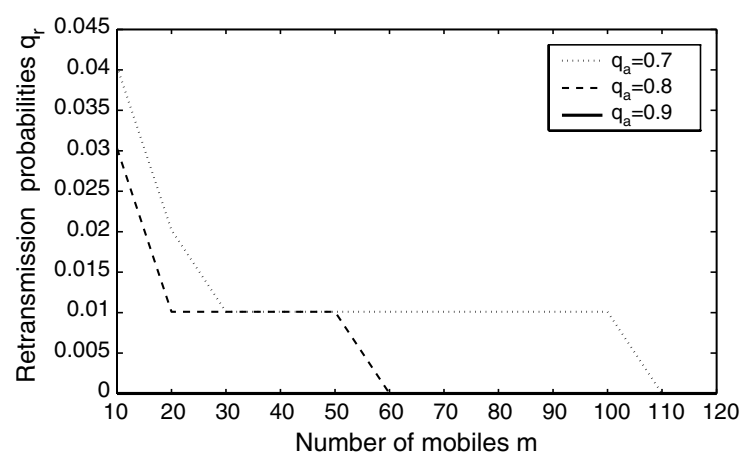

Fig. 4. The optimal retransmission probabilities in the team case as a function of the number of mobiles $m$ for $q_{a}=0.7,0.8$, 0.9 .

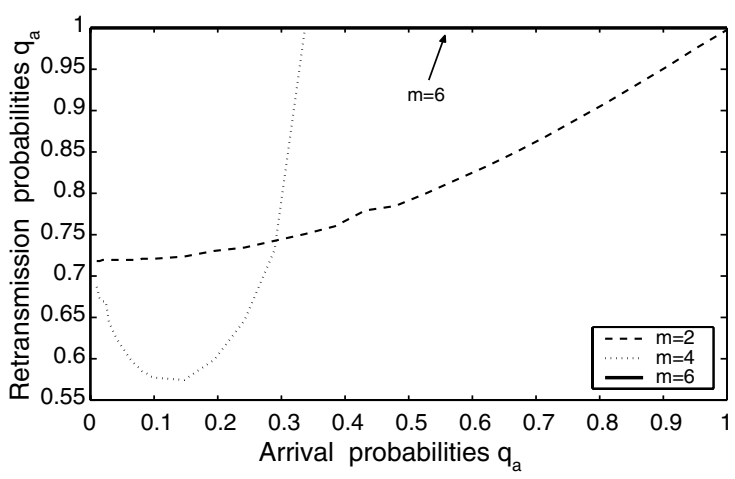

Fig. 6. The optimal retransmission probabilities in the game case as a function of the arrival probabilities $q_{a}$ for $m=2,4,6$. 
resource. For a given symmetric $q$ for all users, the steady-state retransmission cost is $\theta q \sum_{n=0}^{m} \pi_{n}(q) n$, where as the transmission cost of arriving packets (i.e. packets that enter the system and are not rejected) is $\theta \operatorname{thp}(q)$. (This is because the expected number of arrival packets equals to the expected number of departing packets at steady-state, and each time a packet arrives at the system it is immediately transmitted.)

Thus the new team problem is

$$
\max _{q}\left\{\operatorname{thp}(q)(1-\theta)-\theta q \sum_{n=0}^{m} \pi_{n}(q) n\right\} .
$$

For the noncooperative problem, the retransmission cost for a symmetric retransmission policy $q_{r}^{o}$ of users $1, \ldots, m$ and a retransmission probability $q_{r}^{(m+1)}$ of user $m+1$ is

$$
\theta q_{r}^{(m+1)} \sum_{n=0}^{m} \pi_{n, 1}\left(\left[q_{r}^{o}\right]^{-(m+1)}, q_{r}^{(m+1)}\right) .
$$

User $m+1$ is thus faced with the problem:

$$
\max _{q_{r}^{m+1}} J_{m+1}\left(q_{r}^{o}, q_{r}^{(m+1)}\right)
$$

where

$$
J_{m+1}\left(q_{r}^{o}, q_{r}^{(m+1)}\right)=\operatorname{thp}_{m+1}\left(\left[q_{r}^{o}\right]^{-(m+1)}, q_{r}^{(m+1)}\right)(1-\theta)-\theta q_{r}^{(m+1)} \sum_{n=0}^{m} \pi_{n, 1}\left(\left[q_{r}^{o}\right]^{-(m+1)}, q_{r}^{(m+1)}\right) .
$$

Define as we did before

$$
\overline{\mathcal{Q}}_{r}^{m+1}\left(q_{r}^{o}\right)=\operatorname{argmax}_{q_{r}^{(m+1)} \in[\epsilon, 1]}\left(J_{m+1}\left(\left[q_{r}^{o}\right]^{-(m+1)}, q_{r}^{(m+1)}\right)\right) .
$$

Then we seek for the value $q_{r}^{*}$ of retransmission probability that satisfies

$$
q_{r}^{*} \in \overline{\mathscr{Q}}_{r}^{m+1}\left(q_{r}^{*}\right) \text {, }
$$

which is the Nash equilibrium for the game problem.

\section{Numerical investigation}

In this section we obtain the retransmission probabilities which solve the team and the game problems with the extra transmission costs. We shall investigate the dependence of the solution on the value $\theta$.

In Figs. 7-12 we depict the throughput obtained at the optimal solution and the optimal retransmission probabilities, respectively, as a function of the arrival probability, for the team problem with $m=2,10,50$, for various values of $\theta$. We see that both the throughput as well as the retransmission probabilities are monotone decreasing in the cost. This can be expected since retransmissions become more costly with increasing $\theta$. An interesting feature is that for any fixed $\theta \neq 0$, the retransmission probabilities first increase in the arrival probability and then decrease. For $\theta=0$, in contrast, the optimal retransmission probability decreases in the arrival probability (which is natural since congestion in the system increases as $q_{a}$ increases).

Next we consider the game problem with $m=2,10,50$ mobiles.

Figs. 13-18 show the impact of $\theta$ on the total throughput and equilibrium retransmission probability $q_{r}$, as a function of the arrival $q_{a}$. We see that increasing the cost $\theta$ results in decreasing the retransmission probabilities. Furthermore with extra cost, the equilibrium retransmission is more and more small when the cost $\theta$ increases. We see also that indeed the throughput is improved considerably by adding a cost on 


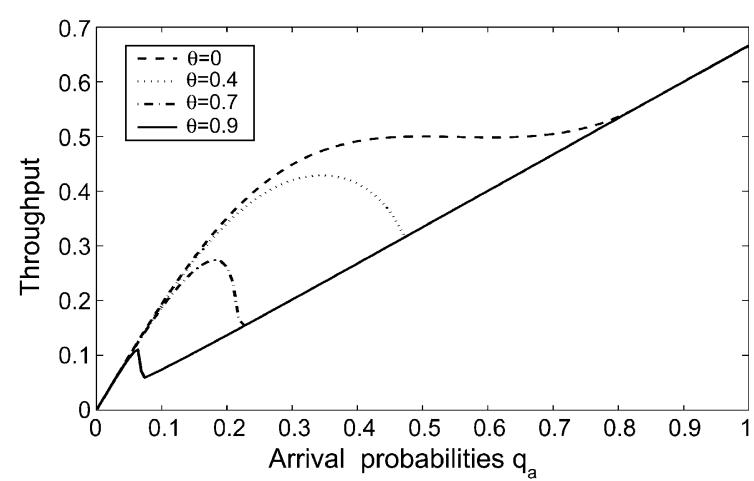

Fig. 7. Throughput at optimal $q_{r}$ for the team case as a function of $q_{a}$ for $m=2$ and $\theta=0,0.4,0.7,0.9$.

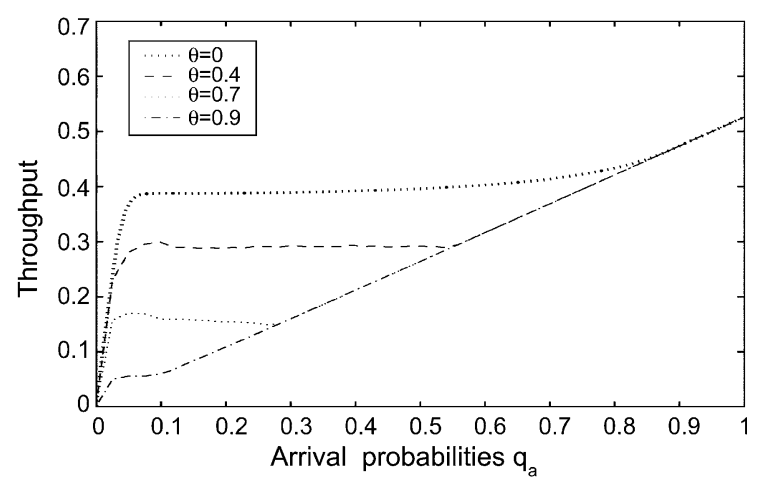

Fig. 9. Throughput at optimal $q_{r}$ for the team case as a function of $q_{a}$ for $m=10$ and $\theta=0,0.4,0.7,0.9$.

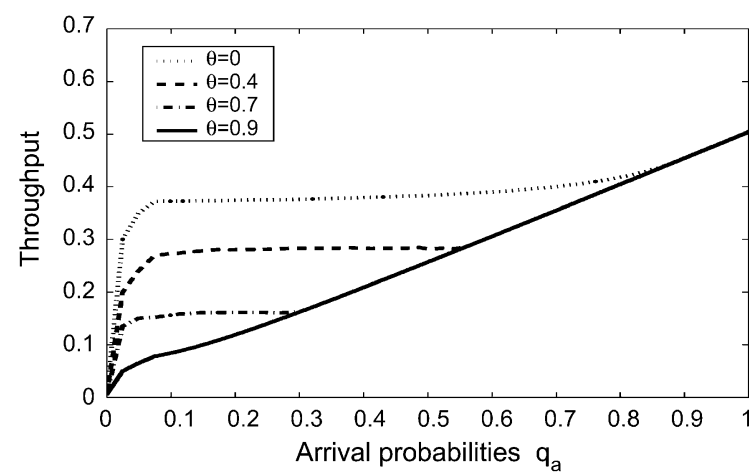

Fig. 11. Throughput at optimal $q_{r}$ for the team case as a function of $q_{a}$ for $m=50$ and $\theta=0,0.4,0.7,0.9$.

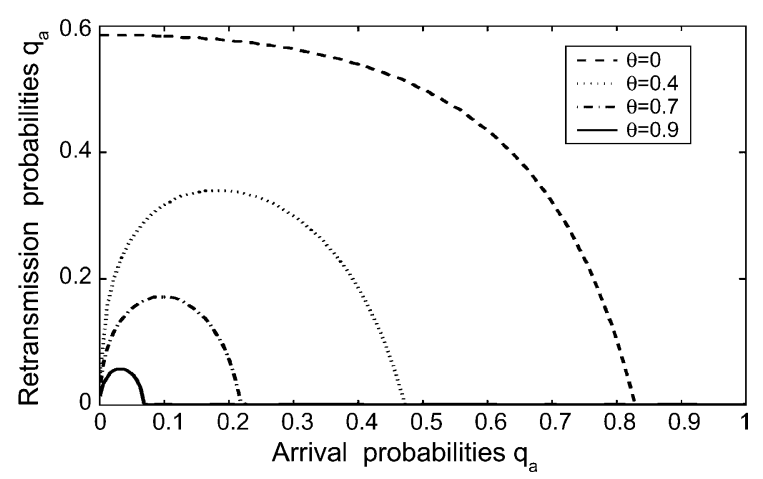

Fig. 8. The optimal retransmission probabilities in the team case as a function of $q_{a}$ for $m=2$ and $\theta=0,0.4,0.7,0.9$.

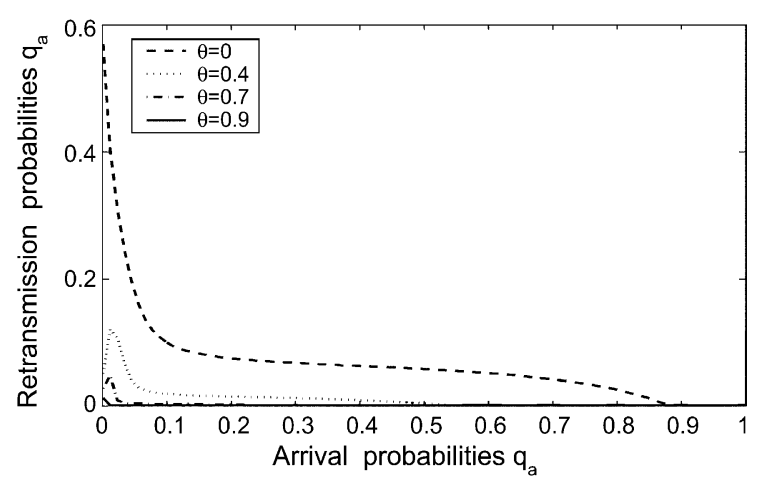

Fig. 10. The optimal retransmission probabilities in the team case as a function of $q_{a}$ for $m=10$ and $\theta=0,0.4,0.7,0.9$.

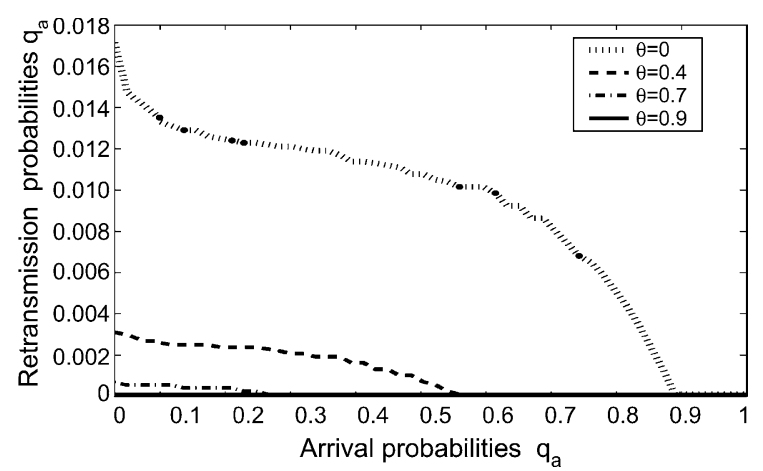

Fig. 12. The optimal retransmission probabilities in the team case as a function of $q_{a}$ for $m=50$ and $\theta=0,0.4,0.7,0.9$. 


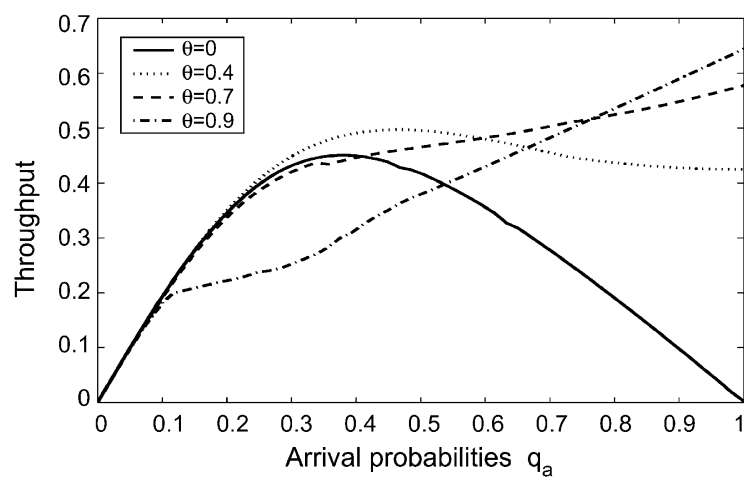

Fig. 13. Total throughput for the game case as a function of the arrival probabilities $q_{a}$ for $m=2$ (number of mobiles) and $\theta=0,0.4,0.7,0.9$.

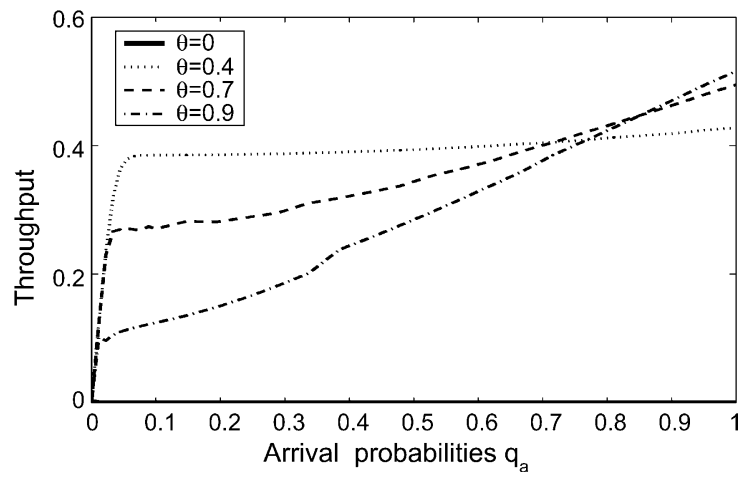

Fig. 15. Total throughput for the game case as a function of the arrival probabilities $q_{a}$ for $m=10$ (number of mobiles) and $\theta=0,0.4,0.7,0.9$.

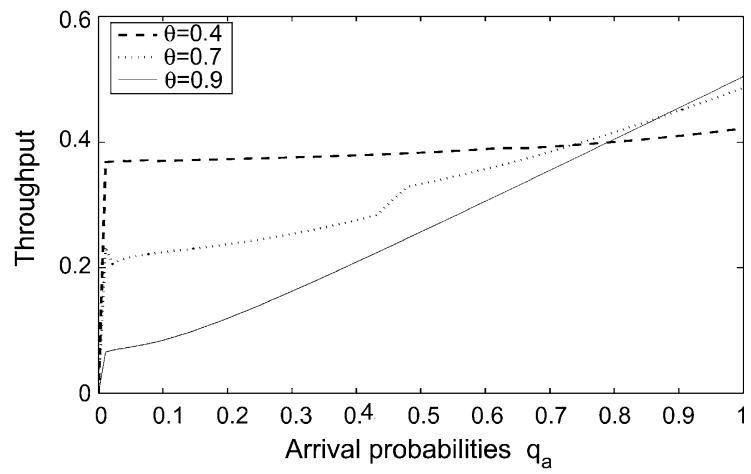

Fig. 17. Total throughput for the game case as a function of the arrival probabilities $q_{a}$ for $m=50$ (number of mobiles) and $\theta=0,0.4,0.7,0.9$.

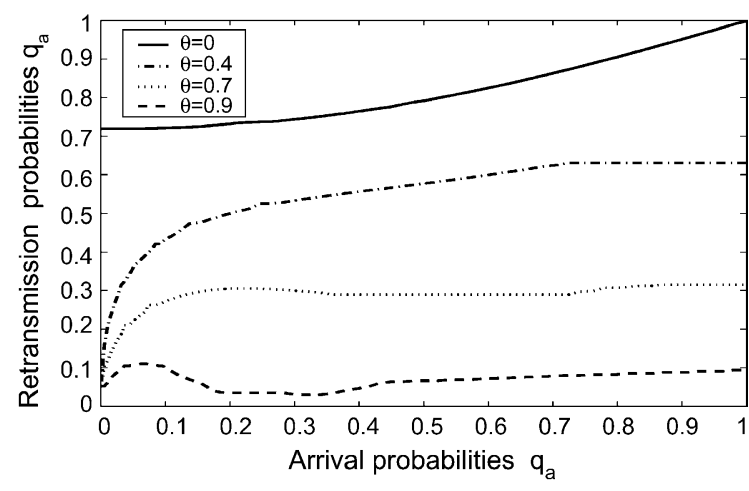

Fig. 14. The equilibrium retransmission probabilities in the game case as function of the arrival probabilities $q_{a}$ for $m=2$ (number of mobiles) and $\theta=0,0.4,0.7,0.9$.

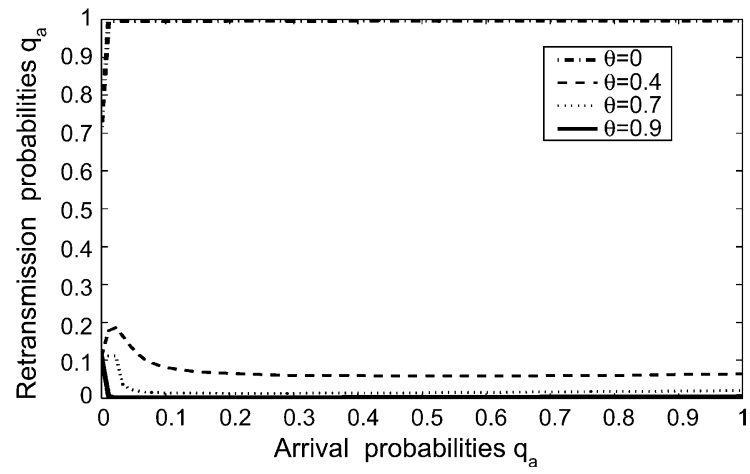

Fig. 16. The equilibrium retransmission probabilities in the game case as function of the arrival probabilities $q_{a}$ for $m=10$ (number of mobiles) and $\theta=0,0.4,0.7,0.9$.

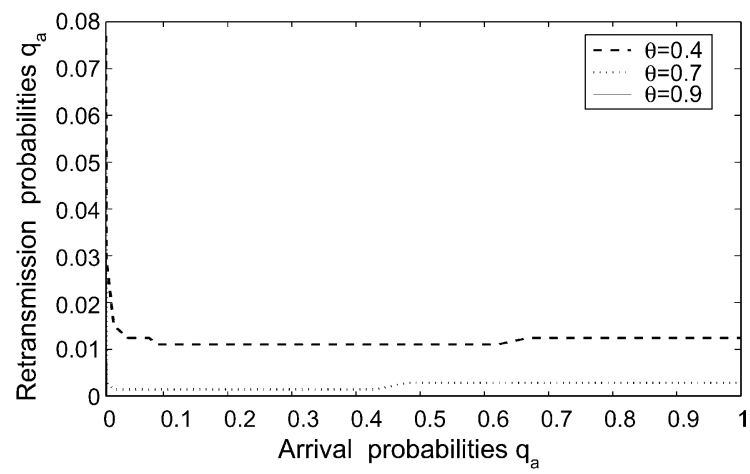

Fig. 18. The equilibrium retransmission probabilities in the game case as function of the arrival probabilities $q_{a}$ for $m=50$ (number of mobiles) and $\theta=0,0.4,0.7,0.9$. 


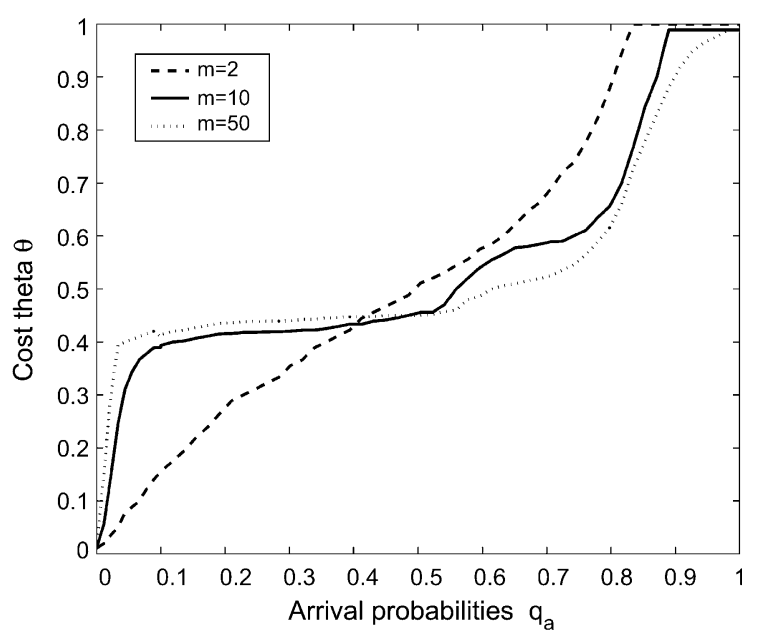

Fig. 19. The retransmission cost $\theta$ such that the optimal retransmission in the game coincides with that of the original team problem, as function of the arrival probabilities $q_{a}$ for $m=2,10,50$.

retransmission, especially for large arrival probabilities or for $m>3$. However, we believe that these bad performances of game problem can be potentially be solved by using this extra cost even with large number of mobiles. We observe also that for different values of $q_{a}$, we obtain different costs $\theta$ which gives best throughput. For example, in Fig. 13, for $q_{a}=0.4, \theta=0.4$ gives best throughput and for $q_{a}=0.9, \theta=0.9$ gives best throughput. We then compute the $\operatorname{cost} \theta$ that is necessary for the equilibrium retransmission probabilities to coincide with those obtained for the team problem. This is the value of $\theta$ that will yield the optimal system throughput. The results are presented in Fig. 19 for $m=2,10$ and 50.

From Fig. 19, we see that as the number of mobiles is large $(\geqslant 10)$, the value of $\theta$ that gives the team solution depends less and less on the number of mobiles. This is an appealing property since it suggests that for a large number of mobiles $(m \geqslant 10)$, we may have a pricing choice $\theta$ that can be chosen in a robust way, and may perform close to the team case even if the number of mobiles change.

\section{Concluding remarks}

We have studied three approaches for choosing retransmission probabilities in a slotted Aloha system. First, we studied the team problem, then the noncooperative game problem. The objective was initially to maximize the throughput. We saw that as the arrival probabilities increased, the behavior of mobiles became more and more aggressive (as compared to the team problem) which resulted in a global deterioration of the system throughput. This is in contrast to the team problem in which throughput increased with the arrival probabilities. We also considered additional costs on transmissions and showed numerically that pricing could be used to enforce an equilibrium whose throughput corresponds to the team optimal solution.

\section{Acknowledgements}

This work was partially supported by the EURO NGI network of excellence as well as by the PRIXNET ARC Inria collaboration grant. 


\section{References}

[1] E. Altman, Applications of Markov decision processes in communication networks: a survey, in: E. Feinberg, A. Shwartz (Eds.), Markov Decision Processes, Models, Methods, Directions, and Open Problems, Kluwer, Dordrecht, 2001, pp. $488-536$.

[2] E. Altman, T. Boulogne, R. El Azouzi, T. Jiménez, L. Wynter, A survey on networking games in telecommunications, Computers and Operations Research, in press. doi:10.1016/j.cor.2004.06.005.

[3] E. Altman, L. Wynter, Equilibrium, games, and pricing in transportation and telecommunication networks, Crossovers between Transportation Planning and Telecommunications 4 (1) (2004) 7-21.

[4] N. Abramson, The Aloha system - another alternative for computer communications, AFIPS Conference Proceedings 36 (1970) 295-298.

[5] D. Bertsekas, R. Gallager, Data Networks, Prentice Hall, Englewood Cliffs, NJ, 1987.

[6] D. Dutta, A. Goel, J. Heidemann, Oblivious AQM and Nash equilibria, IEEE Infocom, 2003.

[7] A. Elcan, Optimal customer return rate for an $M / M / 1$ queueing system with retrials, Probability in the Engineering and Informational Sciences 8 (1994) 521-539.

[8] G. Fayolle, E. Gelenbe, J. Labetoulle, Stability and optimal control of the packet switching broadcast channel, Journal of the Association for Computing Machinery 24 (3) (1977) 375-386.

[9] R. Hassin, M. Haviv, On optimal and equilibrium retrial rates in a busy system, Probability in the Engineering and Informational Sciences 10 (1996) 223-227.

[10] Y. Jin, G. Kesidis, Equilibria of a noncooperative game for heterogeneous users of an ALOHA network, IEEE Communication Letters 6 (7) (2002) 282-284.

[11] A.B. MacKenzie, S.B. Wicker, Selfish users in Aloha: a game theoretic approach, in: Proceedings of the Fall 2001 IEEE Vehicular Technology Conference, 2001.

[12] A.B. MacKenzie, S.B. Wicker, Stability of multipacket slotted Aloha with selfish user users and perfect information, in: Proceedings of IEEE Infocom, San Francisco, 2003.

[13] S. Ramakrishna, J.M. Holtzman, A scheme for throughput maximization in a dual-class CDMA system, IEEE Journal Selected Areas in Communication 16 (1998) 830-844.

[14] L.G. Roberts, Aloha packet system with and without slots and capture, Tech. Rep. Ass Note 8, Stanford Research Institute, Advance Research Projects Agency, Network Information Center, 1972.

[15] J.H. Sarker, M. Hassan, S.J. Halme, Power level selection schemes to improve throughput and stability of slotted ALOHA under heavy load, Computer Communications 25 (2002) 1719-1726.

[16] M. Schwartz, Information, Transmission, Modulation and Noise, third ed., McGraw-Hill, New York, 1980.

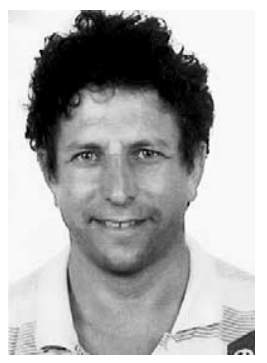

Eitan Altman received the B.Sc. degree in Electrical Engineering (1984), the B.A. degree in Physics (1984) and the Ph.D. degree in Electrical Engineering (1990), all from the Technion-Israel Institute, Haifa. In 1990 he further received his B.Mus. degree in Music Composition in Tel-Aviv university. Since 1990, he has been with INRIA (National research institute in informatics and control) in Sophia-Antipolis, France. His current research interests include performance evaluation and control of telecommunication networks, stochastic control and dynamic games. In recent years, he has applied control theoretical techniques in several joint projects with the French telecommunications company-France Télécom. Since 2000, he has also been with CESIMO, Facultad de Ingeniería, Univesidad de Los Andes, Mérida, Venezuela.

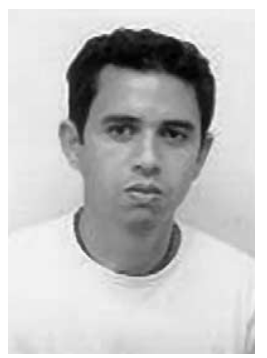

Rachid El Azouzi received the Ph.D. degree in Applied Mathematics from the Mohammed V University, Rabat, Morocco (2000). He joined INRIA (National research institute in informatics and control) SophiaAntipolis for post-doctoral and Research Engineer positions. Since 2003, has been a researcher at the University of Avignon, France. Her research interests are mobile networks, performance evaluation, the TCP protocol, error control in wireless networks, resource allocation, networking games and pricing. 


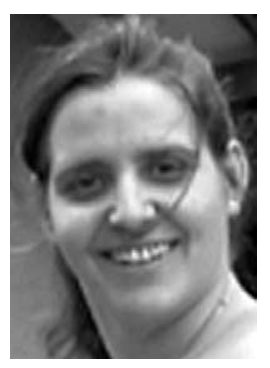

Tania Jiménez received her DEA (equivalent to M.Sc.) at 1997, and Ph.D. at 2000, both in University of Nice Sophia-Antipolis, in Networks and Distributed Systems. Her research interests include simulation as well as optimization and control of telecommunication networks. She has been a teaching and research assistant at Nice university, teaching computer science courses. She is now a lecturer at CESIMO, Facultad de Ingenieria, Universidad de Los Andes, Merida, Venezuela. 